\title{
Realität und Vision der ökologischen Stromversorgung
}

\author{
Internationale Fachtagung der energietechnischen Gesellschaften der electrosuisse, des VDE und der \\ Österreichischen Gesellschaft für Energietechnik im OVE (41. OGE-Fachtagung) am 5. und 6. November \\ 2003 in Salzburg, Salzburg Congress
}

\section{Herzlich Wilkommen in Salzburg!}

Der Blick nach vorne ist die Aufgabe, die wir uns für diese Fachtagung gestellt haben. Die Vorhaben und die ehrgeizigen Ziele der Europäischen Union sowie der einzelnen Staaten in der Energiepolitik wollen erreicht werden. Das geht aber nur, wenn es uns gelingt, die damit verbundenen neuen Herausforderungen aufzuzeigen und zu bewältigen. Deshalb haben wir uns, als die energietechnischen Gesellschaften Deutschlands, der Schweiz und Österreichs, entschlossen, diese Tagung wieder gemeinsam durchzuführen und die notwendigen Voraussetzungen und Bedingungen aufzuzeigen.

Die Richtlinie der Europäischen Union zur Förderung der Stromerzeugung aus erneuerbaren Energiequellen im Energiebinnenmarkt gibt bereits Ziele an, die wir, aus heutiger Sicht beurteilt, wohl nur schwer erreichen werden.

\section{Zur Erinnerung: 12 \% erneuerbare Energie bis 2010.}

Die Voraussetzungen dazu sind entsprechende Entwicklungen im Ausbau der regenerativen Erzeugungskapazitäten unter den Bedingungen der Liberalisierung, aber auch im Lichte sich teilweise konkurrierender EU-Richtlinien bzw. langer Genehmigungswege und im Konsens mit der Bevölkerung. Doch damit ist es noch bei weitem nicht getan. Die Netze müssen für diese Ziele ebenfalls verbessert, verstärkt und angepasst werden, und das bedeutet, dass beachtliche Investitionen zu tätigen und aus technischer, betriebswirtschaftlicher sowie volkswirtschaftlicher Sicht zu bewerten sind. Und ein weiterer Aspekt ist noch gar nicht angeführt: Der Strombedarf steigt ständig, aus heutiger Sicht hoffentlich weiter mit moderaten Zuwachsraten unter $2 \%$.

Die energietechnischen Gesellschaften stehen klar hinter den energiepolitischen Zielen einer umweltfreundlichen und ressourcenschonenden Stromerzeugung. Als Techniker wissen wir auch, wie das System funktioniert. Gerade aus diesem Grund ist es unsere Pflicht darauf hinzuweisen, dass wir die technischen Voraussetzungen einhalten müssen, um die Grundregeln der Stromversorgung sicherstellen zu können. Strom kann nicht gelagert werden, er kann erst dann produziert werden, wenn er gebraucht wird; er funktioniert in einem System, in dem tausende von Stromerzeugern synchron zusammenwirken; der Strom fließt nach einfachen und klaren Gesetzen, die Herr Kirchhoff beschrieben hat, durch Kabel und Leitungen beinahe mit Lichtgeschwindigkeit und als elektromagnetische Welle zum Kunden; er muss da sein, egal von welchem liberalisierten $\mathrm{Er}$ -

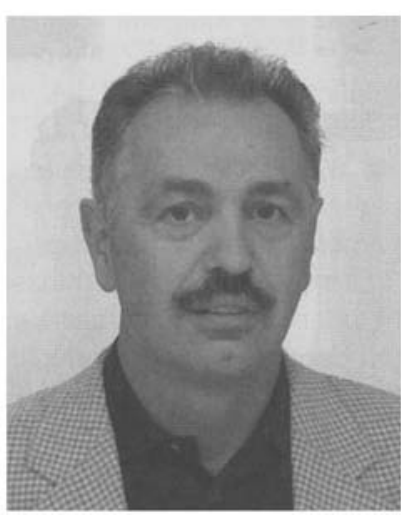

zeuger er produziert, von welchem Übertragungs- und Verteilnetzbetreiber er auch über Regelzonen und Ländergrenzen hinweg übertragen und von welchem Kunden er genutzt wird. Es ist aber für jeden einzelnen wichtig zu wissen, ist zuwenig Strom $\mathrm{da}$, wird es dunkel, es bleibt nur hell, wenn das Zusammenwirken des gesamten Systems funktioniert'. Einen leisen Vorgeschmack gab es bereits im heurigen Sommer.

Die Anforderungen an eine realistische Vision zur ökologischen Stromversorgung, erfahren Sie dazu auf dieser Tagung. In diesem Sinne wünschen wir thnen als Teilnehmer eine interessante und aufschlussreiche Fachtagung sowie einen erfolgreichen und angenehmen Aufenthalt in Salzburg, den Lesern der e \& i viel Freude mit diesem Heft.

Und wegen des Erfolgs haben wir verlängert: Die Vergabe der OGE-Förderpreise 2003 für herausragende Dissertationen, Diplomarbeiten und HTL-Projektarbeiten findet wieder im Rahmen der Fachtagung statt.

Zum Schluss gilt es noch, den Kollegen des Redaktionsteams, den Sponsoren, der Redaktion der e \& I und den Referenten zu danken, die durch ihre Arbeit den Erfolg der Tagung sicherstellen.

\section{Dipl.-Ing. Waiter Auer OVE}

Vorstandsvorsitzender der OGE

\footnotetext{
Die meisten Elektrotechniker sagen sicher: Alles klar, wozu steht das da, aber ist es uns Technikern schon gelungen, das Systemverständnis an Nicht-E-Techniker zu transportieren?
} 